\title{
Estimating the influence of body mass index (BMI) on mortality using offspring BMI as an instrumental variable
}

Elina Hyppönen ${ }^{1,2,3}$, David Carslake ${ }^{4,5}$, Diane J. Berry ${ }^{2}$, Chris Power ${ }^{2}$, George Davey Smith ${ }^{4,5}$

${ }^{1}$ Australian Centre for Precision Health, Unit of Clinical and Health Sciences, University of South Australia, North Terrace, Adelaide, SA 5000, Australia

${ }^{2}$ Population, Policy and Practice, UCL Great Ormond Street Institute of Child Health, 30 Guilford Street, London WC1N 1EH, UK

${ }^{3}$ South Australian Health and Medical Research Institute, North Terrace, Adelaide SA 5000

${ }^{4}$ MRC Integrative Epidemiology Unit at the University of Bristol, Oakfield House, Oakfield Grove, Bristol BS8 2BN, UK

${ }^{5}$ Population Health Sciences, Bristol Medical School, Oakfield House, Oakfield Grove, Bristol BS8 2BN, UK

Correspondence to: Professor Elina Hyppönen, email: elina.hypponen@unisa.edu.au. Australian Centre for Precision Health , c/o SAHMRI, University of South Australia, GPO Box 2471, Adelaide SA 5001, Australia

Words; abstract 245, text 3744 excluding title page, abstract, figures, and tables Keywords: mortality, intergenerational, cohort study, life-course epidemiology, body mass index, obesity, instrumental variable 


\section{ABSTRACT}

\section{Objective}

High body mass index (BMI) is an important predictor of mortality but estimating underlying causality is hampered by confounding and pre-existing disease. Here we use information from the offspring to approximate parental BMIs, with an aim to avoid biased estimation of mortality risk caused by reverse causality.

\section{Methods}

The analyses were based on information on 9674 offspring-mother, and 9096 offspring-father pairs obtained from the 1958 British birth cohort. Parental BMI - mortality associations were analysed using conventional methods, and using offspring BMI as a proxy, or instrument, for their parents' BMI.

\section{Results}

In the conventional analysis, associations between parental BMI and all-cause mortality were U-shaped (Pcurvature $<0.001$ ), while offspring BMI had linear associations with parental mortality $\left(\mathrm{P}_{\text {trend }}<0.001, \mathrm{P}_{\text {curvature }}>0.46\right)$. Curvature was particularly pronounced for mortality from respiratory diseases and from lung cancer. Instrumental variable analyses suggested a positive association between BMI and mortality from all causes [Mothers: HR per SD of BMI 1.43 (95\% CI 1.21 to 1.69$)$. Fathers: HR 1.17, (1.00 to 1.36)] and from coronary heart disease [Mothers: HR 1.65 (1.15 to 2.36). Fathers: HR 1.51, (1.17 to 1.97)]. These were larger than HR from the equivalent conventional analyses, despite some attenuation by adjustment for social indicators and smoking. 


\section{Conclusions}

Analyses using offspring BMI as a proxy for parental BMI suggest that the apparent adverse

consequences of low BMI are considerably overestimated and adverse consequences of overweight are underestimated in conventional epidemiological studies. 


\section{INTRODUCTION}

Obesity is an important risk factor for the leading causes of death, including coronary heart disease (CHD), stroke and several types of cancer $(1,2)$. In observational studies, adult body mass index (BMI) has typically a J- or U-shaped association with subsequent mortality $(3,4)$. It is likely that some of the apparent adverse effect of low adult BMI is due to reverse causality (underlying disease conditions) (5-8), which would also lead to the underestimation of the harmful effects of high adult BMI, and of the public health implications of higher BMI and obesity prevalence in populations. Furthermore, well established confounding factors such as smoking or social circumstances may contribute to the inverse association between BMI and mortality risk at low BMI (8-10). A meta-analysis (11) of studies demonstrating that the optimal BMI with respect to future morality risk appears to be in the overweight range received considerable publicity, with the clear implication of the reporting of this and similar studies being that overweight could have health benefits $(12,13)$.

It is unlikely that clinical trials could provide the answer to the true causal association between BMI and mortality risk (6), hence, there is great interest in exploring epidemiological methods to overcome problems with reverse causality and confounding. A meta-analysis(8) which omitted ever-smokers, those with diagnosed pre-existing disease and the first five years of follow-up as extreme steps to avoid confounding, observed the lowest mortality in the $20-25 \mathrm{~kg}$ $\mathrm{m}^{-2}$ range. The selection criteria and potential bias introduced by focussing on sub-groups included in this study have been criticized (14). Mendelian randomization, in which the subjects' genotypes are used as an instrumental variable for the exposure of interest, is increasingly being used to make unconfounded estimates of the effects of BMI on health outcomes (15-17). In this paper we use an alternative approach, in which offspring BMI is used as a proxy marker for the 
parents' own BMI in an instrumental variables analysis $(18,19)$. The key requirements for an instrumental variable are that it needs to be associated with the exposure of interest, whilst not associated with the outcome (except via the exposure) or with the variables which confound the association between the exposure and the outcome (20). Offspring BMI is a potentially good instrument for parental BMI, as there is a strong correlation in body fatness between parents and their offspring (21), and offspring BMI is independent of underlying disease that may affect the BMI of the parent in most situations. Thus reverse causality, which could have a major impact on the association between own BMI and future mortality, is unlikely to generate distorted associations when offspring BMI is used as an instrumental variable. Confounding by socioeconomic and other risk factors will still exist with the use of offspring BMI, with the greatest bias when the instrument is strongly associated with the potential confounder, but weakly associated with the exposure (22). Furthermore, adverse influences during pregnancy which affect maternal health later in life and which also affect the risk of obesity in her offspring could lead to stronger associations between offspring BMI and mortality risk in mothers compared to fathers (23). This additional association would not be mediated by maternal BMI, and would thus confound the instrumental variables estimates. Such a mechanism is suggested by observations of higher BMIs among offspring to mothers who smoked during pregnancy (24) and individuals who had been exposed to famine in utero (25). However, the causal nature of the association between maternal cigarette smoking and offspring BMI is questionable (24).

We hypothesised 1) that causal associations between BMI and any particular cause of death will be reflected in associations between offspring BMI and parental mortality, and 2) that given the potential influence of the intrauterine environment on maternal associations, the first hypothesis will be better supported for paternal than maternal mortality. We used data 
from two generations in the large nationwide 1958 British birth cohort, with extensive data on social background and smoking, allowing us to evaluate model assumptions and the extent of confounding.

\section{METHODS}

\section{DATA SOURCES}

All births in England, Wales and Scotland in one week, March, 1958 were included in the Perinatal Mortality Survey (coverage 98\%)(26). When the children in the cohort were 7 years old, they were re-contacted and the study continued as the National Child Development Survey (NCDS or 1958 birth cohort). Participants in the cohort have been followed-up regularly thereafter, and detailed information has been collected on growth, health, social and behavioral indicators for the cohort members, their parents and offspring (27).

BMI of parents and offspring was determined as weight $(\mathrm{kg}) /$ height $^{2}\left(\mathrm{~m}^{2}\right)$. Heights and weights of both parents were reported in 1969, usually by the mother. For offspring BMI we used self-reported information collected in 1981 when the offspring were 23 years old; data at that age were more complete and the correlation with parental BMI was slightly stronger compared to other adult ages (28). We used data reported by the mother in 1958 on social class (derived from paternal occupation), maternal smoking during pregnancy and the biological status of the child's mother and father figures. Mother-reported data from 1965 was used for maternal and paternal education and housing tenure. Further smoking data for both parents was reported by the mother in 1974. Cohort members reported their own smoking in 1981 (aged 23) and their educational qualifications in 1991 (aged 33). Parental status was ascertained at the 1965, 1969, and 1974 surveys. If there was any indication that the mother or father figure was not the 
biological parent on any of these occasions, they were classified as "uncertain biological" (29, $30)$.

The methods used to trace the biological parents of all cohort members participating in the first NCDS follow-up in 1965 (target sample 15888 parent pairs) have been previously described in detail $(29,30)$. Parents of English and Welsh cohort members were traced and flagged at the NHS Central Register (NHSCR) at Southport, and parents of Scottish residents were traced through the equivalent register in Edinburgh. The overall tracing rate was $94.9 \%$ for mothers ( $\mathrm{n}=15076)$ and $90.2 \%$ for fathers $(\mathrm{n}=14$ 334) (29). Parental deaths were recorded until $31^{\text {st }}$ December, 2003. Data on the cause of death were coded according to the International Classification of Diseases (ICD, $10^{\text {th }}$ revision). The endpoints used in the study were all-cause mortality (including unknown causes), circulatory diseases (CVD; I01-I99X, G45, G46), coronary heart disease (CHD; I20-I25), stroke (I61-I69, G45, G46, subarachnoid subtype excluded), respiratory diseases (J01-J998A), accidents and violence (S00-Z999), all cancers $(\mathrm{C} 00-\mathrm{C} 97 \mathrm{X})$ and specifically cancers of the lung (C34), breast (C50), prostate (C61), and colon/rectum (C18-C21).

Information was available on offspring BMI in 1981 for $74 \%$ of traced parents, on parents' own BMI in 1969 for $80 \%$ of traced parents and on both for $62 \%$ of traced parents. Data in the final analyses were further restricted to biological parents at risk during the follow-up period, with full information on covariates (Figure S1). The main analyses were thus conducted on 9508 mothers (8 746 fathers) with offspring BMI, 10644 mothers (9 641 fathers) with own BMI, or 8326 mothers (7 638 fathers) with both. 


\section{STATISTICAL ANALYSES}

Parental and offspring BMI were categorised according to sex-specific standard deviation scores (SDS; <-1SDS, -1 to 1 SDS, or >1 SDS). Parental age, smoking and social characteristics were summarised within each category and differences in each characteristic were calculated per 1 SDS increase in parental/offspring BMI using linear or logistic regression as appropriate.

Follow-up for each parent was left-truncated at the approximate date of the offspring's birth (when analysing offspring BMI) or of the parent's BMI measurement (when analysing parental BMI). The follow-up for each parent lasted until death, emigration (245 fathers and 190 mothers), event cancellation (152 fathers and 260 mothers; indicating that a patient had been removed from the doctor's list by the health authority), or the end date for this study ( $31^{\text {st }}$ December, 2003) $(29,30)$. Unadjusted all-cause and cause-specific mortality rates per 1000 person-years at risk were calculated within each category of parental or offspring sex-specific BMI SDS (<-1SDS, -1 to 1 SDS, or >1 SDS) using exponential parametric survival models. Similar models were used to estimate linear and quadratic associations of mortality per 1 SDS increase in BMI.

Hazard ratios for parental mortality per SDS of own and offspring BMI were estimated separately using Cox proportional hazards models with parental age as the time axis. Analyses of maternal and paternal mortality were carried out separately. Schoenfeld residuals for the exposure term were recorded and the P-value from their correlation with the natural logarithm of follow-up time was used to test the proportional hazards assumption. All models were adjusted for parental date of birth as a linear covariate and stratified by offspring sex and a binary variable indicating whether the parent recorded in 1958 was the biological parent. In addition, fully adjusted models controlled for social class in 1958 (Registrar General's classification I \& II, III 
non-manual, III manual, or IV \& V; with households without a male head of household included in the fourth category), the outcome parent's education (stayed at school after minimum leaving age, yes or no), home ownership in 1965 (owner-occupier, private tenant, or council tenant), and maternal smoking during pregnancy (two variables: (i) never, ex-smoker or current smoker and (ii) non-smoker, medium smoker, heavy smoker or variable). In two sensitivity analyses, further adjustment was made for offspring smoking in 1981 (never, ex-smoker, or current smoker) or for paternal smoking in $1974(<1,1-5,6-10,11-20$, or $\geq 21$ cigarettes per day, with pipe or cigar smokers counted in the second category and missing data as a sixth category). The shape of the association between parental all-cause mortality and their own or their offspring's BMI was plotted by repeating the Cox models with the main adjustment set, but with BMI coded into categories of $<18.5,18.5$ to $<20,20$ to $<25,25$ to $<30,30$ to $<35$ and $\geq 35 \mathrm{~kg} \mathrm{~m}^{-2}$.

Estimates of parental hazard ratios per SDS of own BMI were additionally made using offspring BMI SDS as an instrumental variable (IV) (31). This approach avoids some sources of confounding that are present in a conventional observational analysis of own BMI, given certain assumptions(20). To make IV estimates of the hazard ratios, it was first necessary to estimate the association of BMI between the generations. This was done using a linear regression of parental BMI SDS against offspring BMI SDS with each of the adjustment sets described above for the Cox models. For analogy with the stratification of the Cox models, additional adjustment was made in these models for offspring sex and the biological status of the parent registered at birth. IV estimates of the hazard ratios per SDS of own BMI were made by the ratio method, in which the numerators were the natural logarithms of the hazard ratios per SDS of offspring BMI (from the Cox models) and the denominator in each case was the similarly adjusted regression coefficient for parental BMI SDS against offspring BMI SDS. These ratios were exponentiated 
to provide IV estimates of the hazard ratio per SDS of own BMI. Confidence intervals were calculated using Taylor series expansions (32). Each hazard ratio estimated by the IV method was compared with the corresponding conventional observational hazard ratio using Durbin-WuHausman tests (33). Bias component plots $(22,34)$ adjusted for offspring sex and the parent's biological status were used to estimate the relative bias due to various measured covariates in each method. In a further sensitivity analysis, estimates of parental hazard ratio per SDS of own BMI by the conventional and instrumental variables methods were made separately for the parents of sons and the parents of daughters. All statistical analyses were done with Stata, version 14 . 


\section{RESULTS}

Mothers were on average younger than fathers, and the majority (54\%) of them were born in the 1930s (range 1909-1944); whereas 63\% of fathers were born in the 1920s or earlier (range 18901943). The SDs of BMI, used to calculate SDS, were 4.02 in mothers, 3.08 in fathers, 3.19 in daughters and 2.91 in sons and the means were $24.13,24.71,21.98$ and $23.23 \mathrm{~kg} \mathrm{~m}^{-2}$, respectively. There was a strong association between parental BMI in 1969 and offspring BMI at age 23y (unadjusted mean difference in parental BMI SDS per SD of offspring BMI: $0.23,95 \%$ CI $0.21,0.25$ for mothers and $0.19,95 \%$ CI $0.17,0.21$ for fathers, Table 1). Mean differences with standard adjustment (used as denominators in the main IV analyses) were 0.22 (95\% CI: $0.20,0.24)$ for mothers and $0.19(0.17,0.22)$ for fathers and the corresponding F statistics were 435.7 and 291.3, respectively. Values for other adjustment sets were similar (Table S5). High parental and offspring BMI were both associated with shorter education and other indicators of lower family socioeconomic position (Table 1). Parental smoking was associated with reductions in their own BMI, but increased BMI among their offspring. BMI and smoking were not clearly associated among the offspring.

In the main sample for analysing parents' own BMI, the maternal and paternal mortality rates were 8.3 and 16.2 deaths per 1000 person-years, respectively. In the main sample for analysing offspring BMI, they were 6.3 and 11.8, respectively. There was a U-shaped association between parents' own BMI in 1969 and subsequent all-cause mortality, while the associations between offspring BMI and parental mortality were linear (Fig $1 \&$ Table $2 ; \mathrm{P}_{\text {curvature }}<0.001$ for parental and $\mathrm{P}_{\text {curvature }}>0.29$ for offspring $\mathrm{BMI}$ in both maternal and paternal comparisons). Non-linearity in the association between the parent's own BMI and mortality risk was evident for major causes 
of death including circulatory and respiratory diseases (Table 2). Mortality from respiratory diseases and lung cancer was particularly increased for individuals with relatively low BMIs, but mothers with lower BMI (<-1.0 SDS) also appeared to have also a higher incidence of circulatory disease and accidental death. Low offspring BMI was associated with higher incidence of paternal death from accidents and violence, while for other causes there was no evidence for increased mortality risk for parents of offspring with low BMIs.

After adjustment for parental date of birth, a linear positive association was observed between offspring BMI and maternal mortality from circulatory diseases including CHD and stroke, respiratory diseases and lung cancer (Table 3). Offspring BMI was also positively associated with paternal mortality from circulatory diseases, CHD and lung cancer, while there was an inverse association between offspring BMI and paternal (but not maternal) mortality from accidents and violence (HR 0.78, 95\% CI 0.63, 0.97). Most associations between offspring BMI and mortality risk which were seen in unadjusted analyses were attenuated but persisted after adjustment for social indicators and smoking (Table 3). Furthermore, among the mothers there was also an association between offspring BMI and mortality from lung cancer, whilst among the fathers the inverse association between BMI and accidental or violent death was not affected by adjustment for social indicators and smoking. IV ratio analyses confirmed the associations between BMI and parental mortality as estimated in the analyses using offspring BMI only, but gave somewhat stronger associations with all outcomes (for CHD, adjusted HR 1.73, 95\% CI 1.20, 2.48, and HR 1.62, 95\% CI 1.24, 2.12 for mothers and fathers respectively, Table 3). There was a suggestion of non-proportional hazards when breast cancer in mothers was analysed against her own BMI (p-values between 0.008 and 0.014, depending on adjustment set). 
However, among 160 tests for non-proportional hazards in total (10 causes of death, two sexes, four adjustment sets, own or offspring BMI), only 11 had $\mathrm{P}<0.05$ (of which four had $\mathrm{P}<0.01$ and none had $\mathrm{P}<0.005$ ). The bias component plots (Figure S2) indicated that for the measured smoking and socioeconomic variables, the bias had they been omitted from instrumental variables analyses was greater than the bias had they been omitted from the conventional analyses. The evidence for the measured demographic variables was more mixed, and variables indicating reverse causation were not available. There was little variation in IV hazard ratios according to the sex of the offspring used as an instrument (Table S6). 


\section{DISCUSSION}

In two generations of the nationwide British 1958 birth cohort, we compared the associations between the parents' own BMI and their subsequent mortality risk with those observed using offspring BMI as a proxy indicator for parental BMI. As expected from existing literature (3), there was a clearly U-shaped association between the parents' own BMI and their mortality risk. Much of the increased mortality among those with relatively high BMIs was due to cardiovascular diseases, whilst elevated mortality amongst the relatively underweight individuals was largely due to respiratory diseases and lung cancer. In the analyses using offspring information, increasing BMI showed the expected association with excess death from $\mathrm{CHD}$, but no increases were observed for mortality from respiratory diseases or lung cancer for parents of relatively thin individuals. These data suggest that much of the apparent adverse effect of relative leanness observed in earlier studies is likely to be due to reverse causality by underlying disease processes or caused by confounding by factors such as smoking. Our findings also question proposed benefits for being overweight compared to lean $(12,13)$ and support linear increases in mortality by increasing degrees of overweight and obesity.

Our study confirms the known association between obesity and CHD mortality, and provides further support for a positive association between BMI and cancer. Interestingly, a large meta-analysis of prospective observational studies reported an inverse association between BMI and lung cancer (35) while in our IV analyses, in line with published Mendelian randomizations $(36,37)$ and earlier studies using offspring BMI as an instrument $(18,38)$, the association was

positive and fairly strong. A similar contrast was seen in our study in the opposing patterns for lung cancer mortality risk between parental and offspring BMI, which demonstrates the value of instrumental variables in overcoming some of the problems likely to be caused by confounding 
or reverse causality. An interesting finding in our study was the inverse association between BMI and mortality from accidents and violence (including suicidal deaths) seen among fathers, which is in line with observations from a large Norwegian cohort demonstrating a linear decrease in suicide risk with increasing BMI (39). A possible explanation for these observations comes through the link between obesity and insulin resistance, as it has been suggested that a good sensitivity to insulin may be associated with low central serotonin levels (inflicted by altered blood fatty acid composition and tryptophan metabolism) $(40,41)$, which can lead to depression and increased risk of suicide (42).

As proposed earlier, offspring BMI is in many ways a relatively good instrument for parental BMI $(18,38)$. The key benefit from the use of offspring BMI as a proxy for parental BMI is overcoming the problem of reverse causation by underlying disease. This could have been achieved also by using genetic instruments to index differences in individual BMI, an approach which has been increasingly undertaken in relation to various end-points (15-17). A perfect instrument would also enable socioeconomic and behavioral confounding to be overcome, but our results suggest that the association between offspring BMI and parental outcomes is likely to suffer from residual confounding which will need to be considered in the analysis stage.

Other methodological issues need to be considered. Large studies are required when planning to use offspring BMI as a proxy indicator for parental adiposity; increased power is required as offspring BMI is affected by genetic influences from the other parent, lifestyle choices and overall health. Despite the relatively large size of our study, we had only limited power to evaluate cause-specific associations by different types of cancer or to compare sons and daughters as a test of instrument validity. Further, although both the mother and the father may 
contribute to offspring BMI equally through genetic and epigenetic mechanisms, associations between offspring BMI and maternal mortality will further be influenced by exposures during pregnancy which affect both the mothers subsequent health and the propensity of developing obesity in her unborn child [e.g. maternal smoking during pregnancy(24)]. For example, associations of offspring BMI with mortality from respiratory diseases or lung cancer were observed for mothers only in our study, and associations for other causes were generally stronger for mothers compared to fathers. The strong effects of adjustment for maternal smoking during pregnancy suggests that this may partly be related to the effects of maternal metabolism during pregnancy, which in turn determines both the intrauterine environment for her offspring and her own health later in life. However, polymorphisms in mitochondrial DNA have been associated with longevity(43), and it is possible that these same variants could affect adiposity. There are methodological explanations for the observed differences between mothers and fathers; nonpaternity is estimated to be in the range of $2 \%-5 \%$ (44), and the available information on parental BMI relied largely on maternal report, both of which are likely to lead to a greater error in paternal compared to maternal measures.

Our study population is based on two generations participating in the 1958 cohort study. By definition, the analyses were restricted to offspring who survived until BMI measurement at age $23 y$ (and their parents), hence, the parent cohort are healthier on average than the general population $(29,30)$. Despite the limited availability of personal data for the parents, overall tracing rates were generally very high (95\% for the mothers, $90 \%$ for the fathers). It is likely that our findings would apply to most of the population, but generalisation to extreme cases such as severely ill children or parents who die young, should be made cautiously. Any sample 
restriction is likely to affect the health in both generations in the same direction, therefore it is unlikely to introduce bias in the intergenerational associations.

There are strong familial correlations in both the tendency to weight gain and in disease risk. Although susceptibility to obesity is thought to have a strong genetic basis this does not limit the extent to which it can be modified by interventions. In this intergenerational cohort study, we show that while the associations between the parents own BMI and mortality are clearly U-shaped, associations approximated using offspring BMI are linear. Instrumental variables analyses provided no evidence for unfavourable effects by low BMI, suggesting that findings for an elevated mortality risk in previous studies using conventional approaches have most likely reflected underlying disease processes or the effects of smoking. These data suggest that the apparent adverse consequences of low BMI are likely to be overestimated, and adverse consequences of overweight are underestimated in conventional epidemiological studies. Public Health programs should not be based on the potentially misleading conventional observational analyses in this field. 
Contributors: EH, CP and GDS conceived the study. Data analysis was conducted by DC, DJB and EH. The manuscript was written by EH and all authors contributed to its revision and the interpretation of the results. EH had full access to all data in the study.

Funding: The study was funded by the Wellcome trust (ref 059480/Z/99/A). EH was funded by the Department of Health, Public Health Career Scientist Award and statistical analyses the UK Medical Research Council (MRC grant G0601653). This work was supported by the National Institute for Health Research Biomedical Research Centre at Great Ormond Street Hospital for Children NHS Foundation Trust and University College London. The views expressed in the publication are those of the authors and not necessarily those of the Department of Health. GDS and DC work in a unit which receives funds from the UK Medical Research Council (grant numbers 2013-2018: MC_UU_12013/1 and MC_UU_12013/9, 2018-2023: MC_UU_00011/1) and the University of Bristol.

We thank the Centre for Longitudinal Studies (CLS), UCL Institute of Education, for the use of 1958-NCDS data and the UK Data Service for making them available. Neither CLS nor the UK Data Service bear any responsibility for the analysis or interpretation of these data.

Competing interests: All authors have completed the ICMJE uniform disclosure form at www.icmje.org/coi_disclosure.pdf and declare: no support from any organisation for the submitted work; no financial relationships with any organisations that might have an interest in the submitted work in the previous three years; no other relationships or activities that could appear to have influenced the submitted work. 
Ethical approval: Ethical committee approval for the survey at 42 years of age was obtained from the North Thames Medical Research Ethics Committee (MREC), and for the survey at 44 years of age from the South East MREC. Ethical committee approval was not sought for the survey at 33 years of age, although cohort members were asked to give written consent for access to medical records. Ethical approval for the intergenerational research was obtained from the local research ethics committee (Great Ormond Street Hospital/Institute of Child Health, London).

Data sharing: Access to the data from the 1958 British birth cohort can be obtained through the UK DATA Service. https://www.ukdataservice.ac.uk/ 
1. Calle EE, Kaaks R. Overweight, obesity and cancer: Epidemiological evidence and proposed mechanisms. Nature Reviews Cancer. 2004;4(8):579-91.

2. Whitlock G, Lewington S, Sherliker P, Clarke R, Emberson J, Halsey J, et al. Body-mass index and cause-specific mortality in 900000 adults: collaborative analyses of 57 prospective studies. Lancet. 2009;373(9669):1083-96.

3. Flegal KM, Graubard BI, Williamson DF, Gail MH. Cause-specific excess deaths associated with underweight, overweight, and obesity. JAMA. 2007;298(17):2028-37.

4. Winter JE, MacInnis RJ, Wattanapenpaiboon N, Nowson CA. BMI and all-cause mortality in older adults: a meta-analysis. American Journal of Clinical Nutrition. 2014;99(4):875-90.

5. Rose G. The Strategy of Preventive Medicine. Oxford: Oxford University Press; 1992.

6. Seidell JC, Visscher TL, Hoogeveen RT. Overweight and obesity in the mortality rate data: current evidence and research issues. MedSciSports Exerc. 1999;31(11 Suppl):S597-S601.

7. Berrigan D, Troiano RP, Graubard BI. BMI and mortality: the limits of epidemiological evidence. Lancet. 2016;388:734-6.

8. Global BMI Mortality Collaboration. Body-mass index and all-cause mortality: individualparticipant-data meta-analysis of 239 prospective studies in four continents. Lancet. 2016;388:776-86.

9. Joshy G, Korda RJ, Bauman A, Van Der Ploeg HP, Chey T, Banks E. Investigation of methodological factors potentially underlying the apparently paradoxical findings on body mass index and all-cause mortality. Plos One. 2014;9(2):e88641.

10. Patel AV, Hildebrand JS, Gapstur SM. Body mass index and all-cause mortality in a large prospective cohort of white and black US adults. Plos One. 2014;9(10):e109153.

11. Flegal KM, Kit BK, Orpana H, Graubard BI. Association of all-cause mortality with overweight and obesity using standard body mass index categories: A systematic review and meta-analysis. JAMA. 2013;309(1):71-82.

12. Hughes $V$. The big fat truth. Nature. 2013;497:428-30.

13. Kendrick M. Why being "overweight" means you live longer: The way scientists twist the facts. The Independent. $6^{\text {th }}$ April 2015.

14. Flegal KM, Ioannidis JPA, Doehner W. Flawed methods and inappropriate conclusions for health policy on overweight and obesity: the Global BMI Mortality Collaboration meta-analysis. Journal of cachexia, sarcopenia and muscle. 2019;10(1):9-13.

15. Hyppönen E, Mulugeta A, Zhou A, Vimaleswaran KS. A data-driven approach for studying the role of body mass in multiple diseases: a phenome-wide registry-based case-control study in the UK Biobank. Lancet Digital Health. 2019;1(3):11.

16. Wade KH, Carslake D, Sattar N, Davey Smith G, Timpson NJ. BMI and Mortality in UK Biobank: Revised Estimates Using Mendelian Randomization. Obesity (Silver Spring). 2018;26(11):1796-806.

17. Wade KH, Davey Smith G. Adiposity and Cardiometabolic Outcomes: What Can Meta-analyses of Mendelian Randomization Studies Contribute? JAMA network open. 2018;1(7):e183778.

18. Davey Smith G, Sterne JA, Fraser A, Tynelius P, Lawlor DA, Rasmussen F. The association between BMI and mortality using offspring BMI as an indicator of own BMI: large intergenerational mortality study. BMJ. 2009;339:b5043.

19. Cawley J. An instrumental variables approach to measuring the effect of body weight on employment disability. Health ServRes. 2000;35(5 Pt 2):1159-79.

20. Didelez V, Meng S, Sheehan NA. Assumptions of IV methods for observational epidemiology.

Statistical Science. 2010;25(1):22-40.

21. Power C, Pouliou T, Li L, Cooper R, Hypponen E. Parental and offspring adiposity associations: insights from the 1958 British birth cohort. Ann Hum Biol. 2011;38(4):390-9.

22. Jackson JW, Swanson SA. Toward a clearer portrayal of confounding bias in instrumental variable applications. Epidemiology. 2015;26(4):498-504.

23. Davey Smith G. Assessing intrauterine influences on offspring health outcomes: can epidemiological studies yield robust findings? Basic Clin PharmacolToxicol. 2008;102(2):245-56.

24. Power $\mathrm{C}$, Atherton $\mathrm{K}$, Thomas $\mathrm{C}$. Maternal smoking in pregnancy, adult adiposity and other risk factors for cardiovascular disease. Atherosclerosis. 2010;211(2):643-8.

25. Ravelli AC, Der Meulen JH, Osmond C, Barker DJ, Bleker OP. Obesity at the age of $50 \mathrm{y}$ in men and women exposed to famine prenatally. American Journal of Clinical Nutrition. 1999;70(5):811-6.

26. Butler NR, Bonham DG. Perinatal Mortality. Edinburgh: Livingstone; 1963. 
27. Power C, Elliott J. Cohort profile: 1958 British birth cohort (National Child Development Study). International Journal of Epidemiology. 2006;35(1):34-41.

28. Lake JK, Power C, Cole TJ. Child to adult body mass index in the 1958 British birth cohort: associations with parental obesity. ArchDisChild. 1997;77(5):376-81.

29. Hyppönen E, Davey Smith G, Shepherd P, Power C. An intergenerational and lifecourse study of health and mortality risk in parents of the 1958 birth cohort: (I) methods and tracing. Public Health. 2005;119(7):599-607.

30. Hyppönen E, Davey Smith G, Shepherd P, Power C. An intergenerational and lifecourse study of health and mortality risk in parents of the 1958 birth cohort: (II) mortality rates and study representativeness. Public Health. 2005;119(7):608-15.

31. Angrist JD, Krueger AB. The effect of age at school entry on educational attainment: an application of instrumental variables with moments from two samples. Journal of the American Statistical Association. 1992;87(418):328-36.

32. Thomas DC, Lawlor DA, Thompson JR. Re: Estimation of bias in nongenetic observational studies using "Mendelian triangulation" by Bautista et al. Annals of Epidemiology. 2007;17(7):511-3.

33. Greene WH. Econometric Analysis. 7 ed: Pearson; 2012.

34. Davies NM. An even clearer portrait of bias in observational studies? Epidemiology. 2015;26(4):505-

8.

35. Yang Y, Dong J, Sun K, Zhao L, Zhao F, Wang L, et al. Obesity and incidence of lung cancer: A meta-analysis. Int J Cancer. 2013;132(5):1162-9.

36. Gao C, Patel CJ, Michailidou K, Peters U, Gong J, Schildkraut J, et al. Mendelian randomization study of adiposity-related traits and risk of breast, ovarian, prostate, lung and colorectal cancer. International Journal of Epidemiology. 2016;45(3):896-908.

37. Carreras-Torres R, Haycock PC, Relton CL, Martin RM, Davey Smith G, Kraft P, et al. The causal relevance of body mass index in different histological types of lung cancer: A Mendelian randomization study. Scientific Reports. 2016;6.

38. Wade KH, Carslake D, Tynelius P, Davey Smith G, Martin RM. Variation of all-cause and causespecific mortality with body mass index in one million Swedish parent-son pairs: An instrumental variable analysis. PLoS Med. 2019;16(8):e1002868.

39. Magnusson PK, Rasmussen F, Lawlor DA, Tynelius P, Gunnell D. Body mass index is inveresely associated with suicide mortality. A prospective cohort study of over 1 million men. American Journal of Epidemiology. 2006;163 (1):1-8.

40. Frayn KN, Kingman SM. Dietary sugars and lipid metabolism in humans. American Journal of Clinical Nutrition. 1995;62(1 Suppl):250S-61S.

41. Lipsett D, Madras BK, Wurtman RJ, Munro HN. Serum tryptophan level after carbohydrate ingestion: selective decline in non-albumin-bound tryptophan coincident with reduction in serum free fatty acids. Life SciII. 1973;12(2):57-64.

42. Golomb BA, Tenkanen L, Alikoski T, Niskanen T, Manninen V, Huttunen M, et al. Insulin sensitivity markers: predictors of accidents and suicides in Helsinki Heart Study screenees. Journal of Clinical Epidemiology. 2002;55(8):767-73.

43. Niemi AK, Hervonen A, Hurme M, Karhunen PJ, Jylha M, Majamaa K. Mitochondrial DNA polymorphisms associated with longevity in a Finnish population. HumGenet. 2003;112(1):29-33.

44. Macintyre S, Sooman A. Non-paternity and prenatal genetic screening. Lancet. 1991;338:869-71. 


\section{Figure label}

Fig 1. Associations between parental all-cause mortality by offspring BMI in 1981 (age 23y) and own BMI in 1969. (A) Mothers (B) Fathers. Results from Cox models adjusted for parental date of birth, social class in 1958, maternal or paternal education, house ownership (1965) and maternal smoking during pregnancy (1957), and stratified by offspring sex and parental biological status. BMI was categorised as $<18.5,18.5$ to $<20,20$ to $<25,25$ to $<30,30$ to $<35$ and $\geq 35 \mathrm{~kg} \mathrm{~m}^{-2}$ and hazard ratios for each category, relative to a reference level of 20 to $<25$

$\mathrm{kg} \mathrm{m}^{-2}$, were plotted against the mean BMI for that category. 
Table 1. Age, smoking, and social characteristics by parental and offspring BMI.

\begin{tabular}{|c|c|c|c|c|c|c|c|c|c|c|}
\hline \multirow[b]{2}{*}{ Variable } & \multicolumn{5}{|c|}{ Parental BMI SDS in 1969} & \multicolumn{5}{|c|}{ Offspring BMI SDS in 1981 (23 years old) } \\
\hline & $<-1$ & -1 to 1 & $>1$ & Per SD* $(95 \% \mathrm{Cl})$ & $\mathrm{N}+$ & $<-1$ & -1 to 1 & $>1$ & Per SD* $(95 \% \mathrm{Cl})$ & $\mathrm{N}+$ \\
\hline \multicolumn{11}{|c|}{ BMI of Mothers and their offspring; mother's characteristics } \\
\hline Age in 1958 (years) & 26.8 & 27.1 & 28.3 & $0.53(0.43,0.63)$ & 12,042 & 27.6 & 27.3 & 27.2 & $-0.10(-0.20,0.01)$ & 10,940 \\
\hline BMI SDS in 1969 & -1.31 & -0.13 & 1.84 & & 12,042 & -0.28 & -0.03 & 0.43 & $0.23(0.21,0.25)$ & 9,321 \\
\hline Smokers in 1958 & $55.0 \%$ & $39.0 \%$ & $33.0 \%$ & $0.78(0.75,0.81)$ & 11,903 & $35.0 \%$ & $39.0 \%$ & $48.0 \%$ & $1.17(1.13,1.22)$ & 10,805 \\
\hline Smokers in 1974 & $50.0 \%$ & $37.0 \%$ & $33.0 \%$ & $0.83(0.79,0.87)$ & 8,695 & $32.0 \%$ & $37.0 \%$ & $50.0 \%$ & $1.25(1.20,1.31)$ & 8,074 \\
\hline Schooled beyond minimum & $26.0 \%$ & $27.0 \%$ & $17.0 \%$ & $0.80(0.77,0.84)$ & 12,010 & $28.0 \%$ & $27.0 \%$ & $16.0 \%$ & $0.79(0.75,0.83)$ & 10,912 \\
\hline \multicolumn{11}{|c|}{ BMI of Mothers and their offspring; offspring characteristics } \\
\hline BMI SDS in 1981 & -0.28 & -0.04 & 0.40 & $0.23(0.21,0.25)$ & 9,319 & -1.31 & -0.12 & 1.90 & $1.00(0.00,0.00)$ & 10,940 \\
\hline Smokers in 1981 & $49.0 \%$ & $49.0 \%$ & $54.0 \%$ & $1.09(1.04,1.13)$ & 9,471 & $48.0 \%$ & $50.0 \%$ & $53.0 \%$ & $1.03(0.99,1.07)$ & 10,938 \\
\hline Educated to A level or above & $46.0 \%$ & $47.0 \%$ & $38.0 \%$ & $0.85(0.81,0.89)$ & 8,479 & $49.0 \%$ & $48.0 \%$ & $35.0 \%$ & $0.81(0.78,0.85)$ & 8,471 \\
\hline \multicolumn{11}{|c|}{ BMI of Mothers and their offspring; family characteristics } \\
\hline Father's BMI SDS in 1969 & -0.12 & -0.01 & 0.14 & $0.09(0.07,0.10)$ & 11,510 & -0.32 & -0.01 & 0.31 & $0.19(0.17,0.21)$ & 8,999 \\
\hline Social class IV or V in 1958 & $23.0 \%$ & $21.0 \%$ & $31.0 \%$ & $1.21(1.16,1.26)$ & 11,774 & $21.0 \%$ & $21.0 \%$ & $28.0 \%$ & $1.16(1.11,1.21)$ & 10,697 \\
\hline Home owners in 1965 & $43.0 \%$ & $45.0 \%$ & $32.0 \%$ & $0.82(0.79,0.86)$ & 11,003 & $47.0 \%$ & $45.0 \%$ & $34.0 \%$ & $0.83(0.80,0.87)$ & 9,829 \\
\hline \multicolumn{11}{|c|}{ BMI of Fathers and their offspring; father's characteristics } \\
\hline Age in 1958 (years) & 30.4 & 30.2 & 30.4 & $-0.05(-0.16,0.07)$ & 11,059 & 30.8 & 30.3 & 30.3 & $-0.12(-0.24,0.00)$ & 10,238 \\
\hline BMI SDS in 1969 & -1.39 & -0.07 & 1.70 & & 11,059 & -0.32 & -0.01 & 0.31 & $0.19(0.17,0.21)$ & 8,695 \\
\hline Smokers in 1974 & $57.0 \%$ & $44.0 \%$ & $43.0 \%$ & $0.86(0.82,0.90)$ & 7,895 & $42.0 \%$ & $46.0 \%$ & $51.0 \%$ & $1.16(1.11,1.22)$ & 7,445 \\
\hline Schooled beyond minimum & $24.0 \%$ & $25.0 \%$ & $19.0 \%$ & $0.89(0.85,0.93)$ & 9,939 & $29.0 \%$ & $24.0 \%$ & $14.0 \%$ & $0.76(0.72,0.81)$ & 9,024 \\
\hline \multicolumn{11}{|c|}{ BMI of Fathers and their offspring; offspring characteristics } \\
\hline BMI SDS in 1981 & -0.27 & -0.03 & 0.33 & $0.20(0.17,0.22)$ & 8,691 & -1.31 & -0.13 & 1.90 & $1.00(0.00,0.00)$ & 10,238 \\
\hline Smokers in 1981 & $48.0 \%$ & $49.0 \%$ & $50.0 \%$ & $1.03(0.99,1.08)$ & 8,834 & $47.0 \%$ & $49.0 \%$ & $51.0 \%$ & $1.03(0.99,1.07)$ & 10,236 \\
\hline Educated to A level or above & $45.0 \%$ & $49.0 \%$ & $40.0 \%$ & $0.93(0.89,0.97)$ & 7,903 & $50.0 \%$ & $49.0 \%$ & $36.0 \%$ & $0.81(0.78,0.85)$ & 7,986 \\
\hline \multicolumn{11}{|c|}{ BMI of Fathers and their offspring; family characteristics } \\
\hline Mother's BMI SDS in 1969 & -0.06 & -0.02 & 0.19 & $0.09(0.07,0.10)$ & 10,898 & -0.29 & -0.03 & 0.45 & $0.23(0.21,0.25)$ & 8,650 \\
\hline Social class IV or V in 1958 & $21.0 \%$ & $21.0 \%$ & $25.0 \%$ & $1.09(1.04,1.14)$ & 10,838 & $20.0 \%$ & $20.0 \%$ & $28.0 \%$ & $1.17(1.12,1.22)$ & 10,025 \\
\hline Home owners in 1965 & $42.0 \%$ & $45.0 \%$ & $39.0 \%$ & $0.94(0.90,0.98)$ & 10,131 & $49.0 \%$ & $45.0 \%$ & $33.0 \%$ & $0.82(0.78,0.85)$ & 9,202 \\
\hline
\end{tabular}


†Data restricted to biological parents with information on own BMI in 1969, or offspring BMI in 1981, accordingly. 
Table 2. Parental cause-specific mortality rates (per 1000 person-years at risk) by parental and offspring BMI.

\begin{tabular}{|c|c|c|c|c|c|c|c|c|c|c|c|c|}
\hline & \multicolumn{6}{|c|}{ Parental BMI SDS in 1969} & \multicolumn{6}{|c|}{ Offspring BMI SDS in 1981 (23 years old) } \\
\hline & $<-1$ & -1 to 1 & $>1$ & Cases* & $\mathrm{P}_{\text {trend }}{ }^{\dagger}$ & $\mathrm{P}_{\text {curve }}{ }^{\dagger}$ & $<-1$ & -1 to 1 & $>1$ & Cases* & $\mathrm{P}_{\text {trend }}{ }^{\dagger}$ & $P_{\text {curve }}{ }^{\dagger}$ \\
\hline \multicolumn{13}{|l|}{ Mortality in Mothers } \\
\hline All-cause & 9.76 & 7.61 & 11.89 & 3,191 & $<0.001$ & $<0.001$ & 5.84 & 6.16 & 8.04 & 2,977 & $<0.001$ & 0.292 \\
\hline All circulatory & 2.96 & 2.65 & 5.45 & 1,159 & $<0.001$ & 0.066 & 2.16 & 2.11 & 3.34 & 1,062 & $<0.001$ & 0.700 \\
\hline $\mathrm{CHD}$ & 1.52 & 1.33 & 3.30 & 611 & $<0.001$ & 0.246 & 1.29 & 1.11 & 1.84 & 571 & $<0.001$ & 0.767 \\
\hline Stroke & 0.76 & 0.58 & 0.83 & 241 & 0.111 & 0.527 & 0.47 & 0.42 & 0.59 & 210 & 0.034 & 0.218 \\
\hline All respiratory & 1.39 & 0.71 & 0.65 & 297 & 0.003 & 0.009 & 0.59 & 0.56 & 0.81 & 277 & $<0.001$ & 0.993 \\
\hline All cancer & 3.78 & 3.17 & 3.81 & 1,258 & 0.653 & 0.187 & 2.17 & 2.59 & 2.78 & 1,202 & 0.004 & 0.130 \\
\hline Lung & 1.15 & 0.61 & 0.67 & 258 & 0.044 & 0.002 & 0.40 & 0.48 & 0.67 & 232 & $<0.001$ & 0.651 \\
\hline Breast & 0.59 & 0.68 & 0.77 & 258 & 0.551 & 0.159 & 0.36 & 0.55 & 0.64 & 254 & 0.218 & 0.190 \\
\hline Colorectal & 0.24 & 0.31 & 0.26 & 112 & 0.683 & 0.098 & 0.28 & 0.26 & 0.19 & 120 & 0.763 & 0.529 \\
\hline Accidents \& violence & 0.39 & 0.19 & 0.10 & 77 & 0.070 & 0.090 & 0.17 & 0.14 & 0.13 & 68 & 0.867 & 0.982 \\
\hline \multicolumn{13}{|l|}{ Mortality in Fathers } \\
\hline All-cause & 17.70 & 15.55 & 18.04 & 5,131 & 0.254 & $<0.001$ & 11.40 & 11.67 & 13.24 & 4,835 & 0.004 & 0.930 \\
\hline All circulatory & 7.09 & 7.13 & 9.69 & 2,370 & $<0.001$ & $<0.001$ & 5.22 & 5.35 & 6.49 & 2,239 & $<0.001$ & 0.540 \\
\hline CHD & 4.54 & 5.06 & 7.25 & 1,678 & $<0.001$ & $<0.001$ & 3.32 & 3.83 & 4.69 & 1,584 & $<0.001$ & 0.138 \\
\hline Stroke & 1.03 & 0.97 & 0.99 & 310 & 0.499 & 0.898 & 1.04 & 0.72 & 0.81 & 312 & 0.464 & 0.270 \\
\hline All respiratory & 2.92 & 1.43 & 1.17 & 508 & $<0.001$ & $<0.001$ & 1.21 & 1.09 & 1.28 & 461 & 0.640 & 0.450 \\
\hline All cancer & 5.35 & 5.24 & 4.82 & 1,647 & 0.057 & 0.524 & 3.52 & 3.83 & 4.17 & 1,568 & 0.219 & 0.660 \\
\hline Lung & 2.23 & 1.69 & 1.44 & 549 & $<0.001$ & 0.091 & 1.32 & 1.25 & 1.61 & 531 & 0.042 & 0.479 \\
\hline Colorectal & 0.40 & 0.55 & 0.59 & 170 & 0.496 & 0.572 & 0.19 & 0.43 & 0.39 & 163 & 0.778 & 0.143 \\
\hline Prostate & 0.49 & 0.47 & 0.34 & 144 & 0.235 & 0.649 & 0.30 & 0.35 & 0.39 & 143 & 0.697 & 0.845 \\
\hline Accidents \& violence & 0.47 & 0.35 & 0.38 & 118 & 0.664 & 0.636 & 0.39 & 0.28 & 0.25 & 117 & 0.013 & 0.951 \\
\hline
\end{tabular}

*Data restricted to biological parents with information on own BMI in 1969, or offspring BMI in 1981, accordingly.

†P-values are from Wald tests of linear (trend) or quadratic (curve) BMI SDS terms in an unadjusted exponential survival model. 
Table 3. Hazard ratios for parental mortality by offspring BMI in 1981 (age 23y), parents' own BMI in 1969 and offspring BMI treated as an instrumental variable for parental BMI.

\begin{tabular}{|c|c|c|c|c|c|c|c|c|}
\hline & \multicolumn{4}{|c|}{ Minimally adjusted* HR $(95 \% \mathrm{Cl})$ per SDS change in: } & \multicolumn{4}{|c|}{ Adjusted $+\mathrm{HR}(95 \% \mathrm{Cl})$ per SDS change in: } \\
\hline & Offspring BMI & Own BMI & Own BMI, IV & $\mathrm{P}_{\mathrm{DWH}} * *$ & Offspring BMI & Own BMI & Own BMI, IV & $\mathrm{PDWH}^{* *}$ \\
\hline \multicolumn{9}{|l|}{ Mortality in Mothers } \\
\hline All-cause & $1.16(1.13,1.20)$ & $1.09(1.05,1.12)$ & $1.91(1.64,2.22)$ & $<0.001$ & $1.10(1.06,1.14)$ & $1.12(1.08,1.16)$ & $1.56(1.32,1.85)$ & $<0.001$ \\
\hline All circulatory & $1.22(1.15,1.28)$ & $1.25(1.18,1.31)$ & $2.32(1.83,2.94)$ & $<0.001$ & $1.13(1.07,1.20)$ & $1.26(1.19,1.33)$ & $1.74(1.33,2.28)$ & 0.010 \\
\hline $\mathrm{CHD}$ & $1.21(1.13,1.30)$ & $1.34(1.25,1.44)$ & $2.28(1.66,3.12)$ & $<0.001$ & $1.13(1.04,1.22)$ & $1.36(1.26,1.46)$ & $1.73(1.20,2.48)$ & 0.161 \\
\hline Stroke & $1.19(1.05,1.34)$ & $1.01(0.89,1.15)$ & $2.10(1.23,3.57)$ & 0.004 & $1.11(0.97,1.27)$ & $1.04(0.91,1.19)$ & $1.61(0.87,2.97)$ & 0.147 \\
\hline All respiratory & $1.25(1.13,1.38)$ & $0.77(0.67,0.87)$ & $2.62(1.68,4.08)$ & $<0.001$ & $1.12(1.00,1.26)$ & $0.83(0.73,0.96)$ & $1.70(1.02,2.84)$ & 0.002 \\
\hline Accidents \& violence & $1.04(0.82,1.32)$ & $0.77(0.59,1.00)$ & $1.19(0.43,3.29)$ & 0.191 & $0.93(0.69,1.24)$ & $0.73(0.54,0.99)$ & $0.71(0.19,2.63)$ & 0.714 \\
\hline All cancer & $1.11(1.05,1.17)$ & $0.99(0.93,1.05)$ & $1.54(1.22,1.95)$ & $<0.001$ & $1.08(1.02,1.14)$ & $1.02(0.96,1.09)$ & $1.41(1.08,1.84)$ & 0.011 \\
\hline Lung & $1.29(1.16,1.43)$ & $0.85(0.74,0.97)$ & $2.95(1.89,4.63)$ & $<0.001$ & $1.14(1.01,1.28)$ & $0.96(0.83,1.10)$ & $1.79(1.06,3.03)$ & 0.007 \\
\hline Colon/rectum & $0.99(0.83,1.20)$ & $0.93(0.76,1.13)$ & $0.97(0.44,2.15)$ & 0.935 & $1.00(0.82,1.23)$ & $0.93(0.75,1.16)$ & $1.01(0.40,2.53)$ & 0.854 \\
\hline Breast & $1.09(0.97,1.22)$ & $1.03(0.91,1.16)$ & $1.44(0.87,2.38)$ & 0.185 & $1.07(0.94,1.22)$ & $1.02(0.89,1.17)$ & $1.35(0.74,2.46)$ & 0.386 \\
\hline \multicolumn{9}{|l|}{ Mortality in Fathers } \\
\hline All-cause & $1.07(1.05,1.10)$ & $1.02(0.99,1.05)$ & $1.45(1.25,1.68)$ & $<0.001$ & $1.04(1.01,1.07)$ & $1.00(0.97,1.03)$ & $1.20(1.03,1.41)$ & 0.027 \\
\hline All circulatory & $1.10(1.06,1.15)$ & $1.13(1.09,1.18)$ & $1.66(1.34,2.05)$ & $<0.001$ & $1.07(1.03,1.12)$ & $1.12(1.07,1.17)$ & $1.44(1.14,1.81)$ & 0.026 \\
\hline CHD & $1.13(1.08,1.18)$ & $1.18(1.13,1.24)$ & $1.89(1.48,2.42)$ & $<0.001$ & $1.10(1.04,1.15)$ & $1.18(1.12,1.24)$ & $1.62(1.24,2.12)$ & 0.020 \\
\hline Stroke & $1.00(0.90,1.13)$ & $1.05(0.93,1.18)$ & $1.02(0.56,1.85)$ & 0.955 & $0.99(0.87,1.12)$ & $1.01(0.89,1.14)$ & $0.94(0.50,1.79)$ & 0.841 \\
\hline All respiratory & $1.07(0.98,1.17)$ & $0.71(0.64,0.78)$ & $1.42(0.89,2.27)$ & 0.002 & $0.98(0.88,1.09)$ & $0.69(0.62,0.77)$ & $0.90(0.53,1.55)$ & 0.276 \\
\hline Accidents \& violence & $0.78(0.63,0.97)$ & $1.05(0.87,1.26)$ & $0.28(0.09,0.84)$ & 0.011 & $0.72(0.56,0.91)$ & $0.94(0.76,1.15)$ & $0.18(0.05,0.64)$ & 0.006 \\
\hline All cancer & $1.06(1.01,1.11)$ & $0.95(0.91,1.00)$ & $1.35(1.05,1.75)$ & 0.006 & $1.02(0.97,1.08)$ & $0.93(0.88,0.98)$ & $1.10(0.83,1.46)$ & 0.264 \\
\hline Lung & $1.11(1.03,1.20)$ & $0.85(0.78,0.94)$ & $1.73(1.14,2.63)$ & $<0.001$ & $1.05(0.96,1.15)$ & $0.83(0.75,0.91)$ & $1.28(0.81,2.04)$ & 0.064 \\
\hline Colon/rectum & $1.00(0.85,1.17)$ & $1.06(0.91,1.23)$ & $0.98(0.43,2.23)$ & 0.828 & $0.89(0.74,1.07)$ & $1.03(0.87,1.21)$ & $0.54(0.21,1.39)$ & 0.160 \\
\hline Prostate & $1.09(0.92,1.28)$ & $0.90(0.75,1.08)$ & $1.53(0.67,3.52)$ & 0.206 & $1.09(0.91,1.30)$ & $0.87(0.72,1.06)$ & $1.53(0.61,3.84)$ & 0.232 \\
\hline
\end{tabular}

*Cox models adjusted for parental date of birth and stratified by offspring sex and parental biological status.

${ }^{\dagger}$ Cox models additionally adjusted for social class in 1958, maternal or paternal education, house ownership (1965) and maternal smoking during pregnancy (1957) and stratified by offspring sex and parental biological status.

¥Ratio method estimates using offspring BMI in 1981 (23 years old) as an instrument for parents' own BMI in 1969.

**P value from a Durbin-Wu-Hausman test comparing the conventional and IV HR per SDS change in own BMI.

The largest possible sample was used for each analysis; for offspring BMI, own BMI and separately for the numerator and denominator of the IV analysis. 